\title{
Phylogenetic analysis of infectious bursal disease virus isolated from three provinces of Sri Lanka
}

\author{
RMIM Rathnayake, SSS de S Jagoda, AW Kalupahana
}

Introduction and Objectives: Infectious bursal disease (IBD) is a highly contagious, immunosuppressive disease of young chicken. Affected birds manifest with depression, ruffled feathers, diarrhoea, and reduced appetite. The causative agent of IBD is infectious bursal disease virus (IBDV) of the genus Avibirnavirus in the familyBirnaviridae. IBDV genome consists of two segments of double stranded RNA that encodes five viral proteins, VP1 to VP5. Out of two IBDV serotypes, only serotype 1 is pathogenic in chicken, which is further classified into three pathotypes. More recent classification categorizes IBDVs into seven genogroups. The objective of this study was to sequence the currently circulating IBDVs in Sri Lanka and to determine their genogroups through a phylogenetic analysis.

Methods: Bursa of Fabricius was collected from birds manifesting clinical signs suggestive of IBD from six poultry layer farms in North Western, Central and Sabaragamuwa provinces. Total RNA was extracted, and conventional reverse transcriptase PCR performed targeting the hypervariable region of the VP2 gene. The amplified PCR products (474bp) were purified and sequenced directly. Nucleotide sequences from both strands were aligned and manually edited using MEGA-X. Resulting consensus sequences were analyzed with NCBI BLASTn(http://www.ncbi.nlm.nih.gov/blastn) to search for best matches. Nucleotide sequences from this study and thirteen reference and/or published strains representing seven genogroups of IBDV were aligned using MUSCLE algorithm (MEGA-X). Phylogenetic analysis using 442 nucleotides length of partial VP2 gene was performed using the maximum likelihood method with Kimura two-parameter evolutionary mode. Robustness of the topology of the phylogenetic tree was analyzed by the bootstrap method with 1000 replicates. Bootstrap values lower than $75 \%$ were considered non-significant.

Results:All six samples were positive for IBDV by RT-PCR. In BLASTn analysis, the sample from the Central Province showed the highest sequence similarity (97.5\%) to an IBDV isolate from India (Y18612). Samples from other two provinces showed highest sequence homologies (ranging from 96.8-97.7\%) to Iranian field IBDV isolates (DQ630452 and DQ630448).

Conclusions: Maximum likelihood phylogenetic tree showed a distinct clustering of seven genogroups with all our IBDV sequences clustering into the largest genogroup 3 (G-3) clade.

Keywords: IBDV, VP2 gene, IBDV genogroups, IBDV serotypes

Department of Veterinary Pathobiology, Faculty of Veterinary Medicine and Animal Science, University of Peradeniya, Sri Lanka

Address for correspondence:Dr. AWKalupahana. Telephone: +94713858581

Email: anilwkalupahana@yahoo.com (D) https://orcid.org/0000-0002-9804-3345 